\title{
I-ROAD could be efficient in predicting severity of community-acquired pneumonia or healthcare- associated pneumonia
}

Ryo Matsunuma ${ }^{1}$, MD, Nobuhiro Asai ${ }^{1}$, MD, Yoshihiro Ohkuni ${ }^{1}$, MD, PhD, Kei Nakashima ${ }^{1}$,MD, Takuya Iwasaki ${ }^{1}$, MD, Masafumi Misawa ${ }^{1}, \mathrm{MD}, \mathrm{PhD}$, Norihiro Kaneko' ${ }^{1}$ MD, PhD

INTRODUCTION The ability to predict the prognosis of patients with pneumonia is critical, especially when making decisions regarding treatment regimens and sites of care. However, prognostic guidelines for healthcare-associated pneumonia (HCAP) have yet to be established. I-ROAD is the prognostic guideline of the Japanese Respiratory Society for hospital-acquired pneumonia (HAP). This study compared available prognostic guidelines to determine the usefulness of I-ROAD as a prognostic tool for patients with HCAP.

METHODS We conducted a retrospective review of all patients with pneumonia admitted to Kameda Medical Center, Japan, from January 2006 to September 2009. Patients were categorised into two groups, namely those with communityacquired pneumonia (CAP) and those with HCAP. We compared the baseline characteristics, laboratory findings, identified pathogens, antibiotic regimens, clinical outcomes, pneumonic severity and prognostic accuracy of each guideline between the two patient groups. The severity of each disease was assessed on admission using the A-DROP, CURB-65, PSI and I-ROAD guidelines.

RESULTS Of the 302 patients evaluated, 228 (75.5\%) were diagnosed with CAP and 74 (24.5\%) with HCAP. Patients with HCAP were older and had a higher performance status than patients with CAP. The mortality rate in the CAP group tended to rise with increasing severity scores of prognostic guidelines. Although the severity scores of all prognostic guidelines could predict 30-day mortality in patients with CAP, I-ROAD exhibited a higher discriminatory power for patients with HCAP based on analysis of receiver-operating characteristic curves.

CONCLUSION I-ROAD could be more accurate than other prognostic guidelines for evaluating the severity of HCAP.

Keywords: community-acquired pneumonia, healthcare-associated pneumonia, I-ROAD, prognostic tools, severity

\section{INTRODUCTION}

Pneumonia remains a leading cause of death worldwide. There are several widely used prognostic guidelines issued for communityacquired pneumonia (CAP), namely A-DROP, developed by the Japanese Respiratory Society (JRS); ${ }^{(1)}$ CURB-65, from the British Thoracic Society (BTS);(2) and pneumonia severity index (PSI), from the American Thoracic Society/Infectious Disease Society of America (ATS/IDSA). ${ }^{(3)}$ These three guidelines help clinicians make rational decisions regarding the best treatment regimen and site of care for patients.

Healthcare-associated pneumonia (HCAP) is a new category of respiratory infections documented in the 2005 ATS/IDSA guideline. ${ }^{(4)}$ Patients with HCAP are described as an independent group of patients with pneumonia who are excluded from the CAP category because the epidemiologic pattern is similar to that of hospital-acquired pneumonia (HAP). Although the concept of HCAP has been defined, there are few established prognostic guidelines for HCAP. In 2006, JRS produced I-ROAD, a guideline for HAP. ${ }^{(5,6)}$ I-ROAD is completely different from conventional CAP guidelines in that it uses chest radiographs and serum C-reactive protein (CRP) to categorise disease severity. We conducted this retrospective study to evaluate which prognostic tool is able to more accurately reflect the severity of CAP and
HCAP, and to determine whether I-ROAD is a better predictor of 30-day mortality than the other guidelines.

\section{METHODS}

We retrospectively reviewed the records of all patients with pneumonia admitted to Kameda Medical Center, Japan, from 1 January 2006 to 30 September 2009. The patients were categorised into either the CAP or HCAP group. The severity of both CAP and HCAP were assessed on admission using A-DROP, CURB-65, PSI and I-ROAD. We compared the baseline characteristics, laboratory findings, identified pathogens, antibiotic regimens, clinical outcomes, pneumonic severity and prognostic accuracy of each guideline in each group. The study protocol was approved by the Institutional Review Board of Kameda Medical Center.

A diagnosis of pneumonia requires the presence of a new radiographic infiltrate and at least one of the following: (a) symptoms of infection, such as the presence of a fever $\left(>38.0^{\circ} \mathrm{C}\right)$, productive cough, purulent sputum from the lower respiratory tract, or chest pain; or (b) a white blood cell count $>10,000 / \mu \mathrm{L}$ and/or CRP elevated above $0.03 \mathrm{mg} / \mathrm{dL}$. HCAP and CAP were defined based on ATS/IDSA guidelines. ${ }^{(4)}$ CAP was defined as a diagnosis of pneumonia in patients who did not meet any of the criteria for

${ }^{1}$ Department of Pulmonology, Kameda Medical Center, Chiba, Japan

Correspondence: Dr Ryo Matsunuma, Senior Resident, Department of Pulmonology, Kameda Medical Center, 929 Higashi-cho, Kamogawa City, Chiba Prefecture, Japan 296-8602. manutsuma@gmail.com 


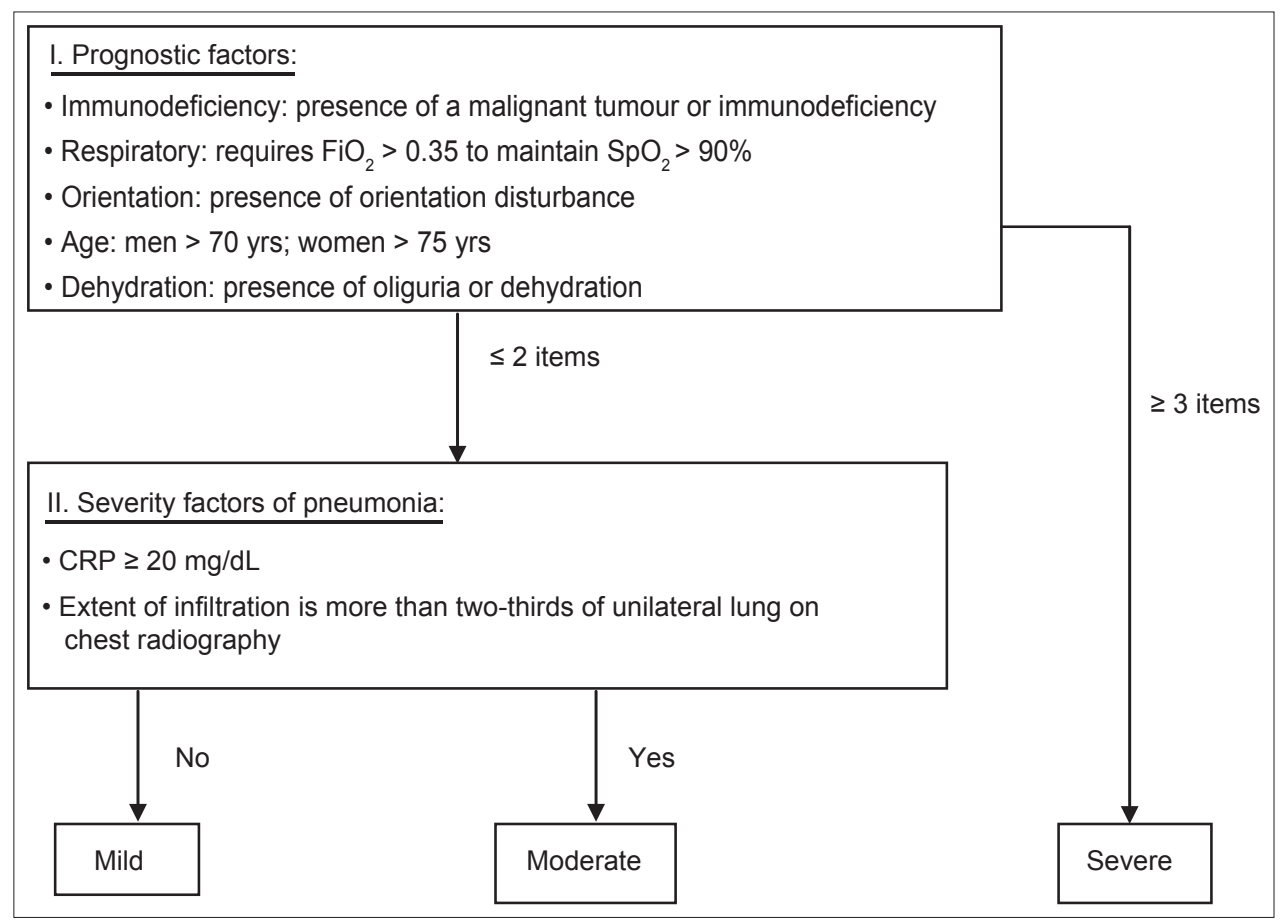

Fig. 1 Flowchart of the I-ROAD prognostic guideline for hospital-acquired pneumonia.

HCAP, i.e. these patients: (a) reside in their own homes and not in a nursing home or hospital; (b) receive no care (i.e. no clinic visits, infusion or haemodialysis); and (c) were not admitted to a hospital within the preceding 90 days. In contrast, HCAP included patients with any of the following: (a) hospitalisation for two days in the preceding 90 days; (b) residence in a nursing home or extended care facility; (c) receiving home infusion therapy (including antibiotics); (d) on long-term dialysis (including haemodialysis and peritoneal dialysis) within the 30 days prior to enlistment into the study; and (e) received home wound care. ${ }^{(4)}$ Patients who visited outpatient clinics were placed in the CAP group instead of the HCAP group, as their general condition is usually similar to that of healthy people.

Initial treatment failure was defined as death during initial treatment or a change in the antibiotic regimen from the initial agents within 72 hours of the start of treatment due to a lack of response or clinical deterioration (e.g. worsening fever, respiratory condition or radiologic status; requiring mechanical ventilation, aggressive fluid resuscitation or vasopressors).

Baseline demographic information such as age, gender, residence (i.e. nursing home or non-nursing home) and performance status (measured using the Eastern Cooperative Oncology Group scale $\left.{ }^{(7)}\right)$, as well as the clinical data of all enrolled patients, were collected through the review of patient medical records. Physical examination findings (i.e. pulse rate, respiratory rate, systolic and diastolic blood pressure [BP], temperature and mental status), the presence of comorbidities (i.e. chronic obstructive pulmonary disease, bronchial asthma, diabetes mellitus, hypertension, dyslipidaemia, collagen vascular disease, neoplastic disease, liver disease, congestive heart failure, cerebrovascular disease and renal disease), and laboratory and radiographic results of the patients were reviewed via the electronic medical records. The source of knowledge of comorbidities was classified as patient declaration or medication record notation. All-cause mortality was assessed for all patients 30 days after the initial presentation, using patient interviews and medical record review.

Pneumonic severity was evaluated using A-DROP, CURB-65, PSI and I-ROAD. A-DROP is a 6-point scoring system proposed by $\mathrm{JRS}^{(1)}$ that assesses the following parameters: (a) age (men $>70$ years, women $>75$ years); (b) dehydration (blood urea nitrogen $[\mathrm{BUN}$ ] concentration $>21 \mathrm{mg} / \mathrm{dL}$ ); (c) respiratory failure (pulse oximetric saturation $<90 \%$, partial pressure of oxygen in arterial blood $\left[\mathrm{PaO}_{2}\right]<60 \mathrm{mmHg}$, or $\mathrm{PaO}_{2}$ /fraction of inspired oxygen ratio $\left[\mathrm{FiO}_{2}\right]<300$ ); (d) orientation disturbance (i.e. confusion); and (e) low BP (systolic BP $<90 \mathrm{~mm} \mathrm{Hg}$ ). Proposed by BTS, ${ }^{(2)}$ CURB-65 evaluates the following parameters: (a) confusion; (b) BUN > $20 \mathrm{mg} / \mathrm{dL}$; (c) respiratory rate $>30$ breaths/min; (d) systolic BP $<90 \mathrm{mmHg}$ or diastolic BP $<60 \mathrm{mmHg}$; and (e) age > 65 years. The PSI, a predictive index proposed by ATS/IDSA, ${ }^{(3)}$ evaluates the following parameters: (a) age > 65 years; (b) residence in a nursing home; (c) coexisting illnesses (e.g. neoplastic disease, liver disease, congestive heart failure, cerebrovascular disease and renal disease); (d) physical examination findings (e.g. altered mental status, respiratory rate $>30 / \mathrm{min}$, systolic $\mathrm{BP}<90 \mathrm{mmHg}$, temperature $<35^{\circ} \mathrm{C}$ or $>40^{\circ} \mathrm{C}$ and pulse 125 beats/min); and (e) laboratory and radiographic findings (arterial $\mathrm{pH}<7.35, \mathrm{BUN}>30 \mathrm{mg} / \mathrm{dL}$, sodium $<130 \mathrm{mEq} / \mathrm{L}$, glucose $>250 \mathrm{mg} / \mathrm{dL}$, haematocrit $<30 \%$, $\mathrm{PaO}_{2}<60 \mathrm{~mm} \mathrm{Hg}$ or oxygen saturation $\left[\mathrm{SpO}_{2}\right]<90 \%$, and pleural effusion). The I-ROAD system, also proposed by JRS, ${ }^{(5,6)}$ Classifies severity of pneumonia according to the number of prognostic factors that apply to a patient (Fig. 1). When three or more prognostic factors apply, the patient is classified as severe. If less 
than three items apply, the patient would be further evaluated to determine whether the CRP level is greater than $20 \mathrm{mg} / \mathrm{dL}$ and whether lung infiltration is more than two-thirds unilateral on chest radiography - a patient who is positive for at least one of the two aforementioned characteristics is deemed to have moderately severe HAP. If none of the two applies, the patient is deemed to have a mild case of pneumonia.

Acceptable positive culture specimens included those obtained from sputum, tracheal aspirate or blood. A positive blood culture was accepted as a true positive if the same microorganism was identified in the respiratory specimen and no other source for the positive blood culture could be identified. In the present study, antimicrobial susceptibility testing was performed using broth microdilution and interpreted according to the guidelines set by the Clinical and Laboratory Standards Institute (CLSI). The susceptibility of Streptococcus pneumoniae strains were evaluated by assessing the minimal inhibitory concentrations, as described in the CLSI guideline. A breakpoint was applied based on the CLSI 2006 guideline, which was published in May 2008. ${ }^{(8)}$

Susceptibility to the following drugs was tested for all recovered microorganisms: amoxicillin, piperacillin sodium, cefotaxime sodium, ceftazidime, cefepime hydrochloride, ciprofloxacin hydrochloride, imipenem, aztreonam, gentamicin sulfate, amikacin sulfate, and a combination of sulfamethoxazole and trimethoprim. Multidrug-resistant (MDR) pathogens included methicillin-resistant Staphylococcus aureus (MRSA), Stenotrophomonas maltphilia and extended spectrum $\beta$-lactamase (ESBL)-producing Enterobacteriaceae, based on previous reports that showed problematic outcomes for infections caused by these pathogens. ${ }^{(9,10)}$

The death rate was calculated for both the CAP and HCAP groups, with low to severe scores based on each set of guidelines. Correlation between the severity score and the accuracy of the predicted 30-day mortality was used to determine the sensitivity, specificity, and positive and negative predictive values of each set of guidelines for the two patient groups. Finally, receiveroperating characteristic (ROC) curves were constructed to plot sensitivity and false positives (i.e. 1-sensitivity) in order to compare the availability of each set of guidelines in patients with CAP and those with HCAP. Normally and non-normally distributed continuous variables were compared using Student's $t$-test and Wilcoxon rank-sum test, respectively. Either chi-square or Fisher's exact test was used to compare categorical variables. A p-value of $\leq 0.05$ was considered statistically significant. All statistical comparisons were done using the Statistical Package for the Social Sciences for Windows version 20.00 (IBM Corp, Armonk, NY, USA).

\section{RESULTS}

The characteristics of the patients enrolled in the present study are shown in Table I. A total of 302 patients were evaluated during the study period - 228 patients with CAP $(75.5 \%)$ and 74 with
Table I. General characteristics of the patients $(n=302)$.

\begin{tabular}{|c|c|c|}
\hline \multirow[t]{2}{*}{ Characteristic } & \multicolumn{2}{|c|}{ No. of patients (\%) } \\
\hline & $\begin{array}{c}\text { CAP } \\
(n=228)\end{array}$ & $\begin{array}{c}\text { HCAP } \\
(n=74)\end{array}$ \\
\hline 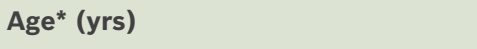 & $74 \pm 13$ & $80 \pm 11$ \\
\hline \multicolumn{3}{|l|}{ Gender } \\
\hline Male & $164(72.0)$ & $46(62.2)$ \\
\hline Female & $64(28.1)$ & $28(37.8)$ \\
\hline Performance status* & $0.1 \pm 0.4$ & $1.4 \pm 1.5$ \\
\hline 30-day mortality rate* & $23(10.1)$ & $14(19.0)$ \\
\hline Treatment failure* & $12(5.3)$ & $1(1.4)$ \\
\hline Residence in a nursing home* & - & $39(52.7)$ \\
\hline Hospitalised within 90 days* & - & $10(13.5)$ \\
\hline Receiving home care* & - & $25(33.8)$ \\
\hline On haemodialysis* & - & $8(10.8)$ \\
\hline \multicolumn{3}{|l|}{ Presence of comorbidity* } \\
\hline Hypertension & $104(45.6)$ & $38(51.4)$ \\
\hline Diabetes mellitus & $38(16.7)$ & $10(13.5)$ \\
\hline Dyslipidaemia & $28(12.3)$ & $10(13.5)$ \\
\hline Collagen vascular disease & $7(3.1)$ & $2(2.7)$ \\
\hline Malignant tumour & $43(18.9)$ & $7(9.5)$ \\
\hline Liver disease & $13(5.7)$ & $7(9.5)$ \\
\hline Chronic heart failure & $29(12.7)$ & $19(25.7)$ \\
\hline Cerebrovascular disease & $39(17.1)$ & $40(54.1)$ \\
\hline Chronic respiratory failure & $22(9.6)$ & $12(16.2)$ \\
\hline Home oxygen therapy & $15(6.6)$ & $11(14.9)$ \\
\hline Chronic kidney disease & $17(7.5)$ & $14(18.9)$ \\
\hline \multicolumn{3}{|l|}{ Laboratory findings* } \\
\hline Haematocrit (\%) & $37 \pm 6.0$ & $35 \pm 4.7$ \\
\hline BUN (mg/dL) & $21 \pm 11$ & $29 \pm 18$ \\
\hline Sodium (mEq/L) & $137 \pm 3.8$ & $137 \pm 5.3$ \\
\hline Glucose (mg/dL) & $145 \pm 53$ & $129 \pm 57$ \\
\hline $\mathrm{CRP}(\mathrm{mg} / \mathrm{dL})$ & $12.9 \pm 9.9$ & $10.2 \pm 7.8$ \\
\hline Arterial pH & $7.42 \pm 0.07$ & $7.41 \pm 0.06$ \\
\hline $\mathrm{PaO}_{2}(\mathrm{mmHg})$ & $83 \pm 31$ & $80 \pm 30$ \\
\hline Lung infiltration $>2 / 3$ on radiography & $66(28.9)$ & $8(10.8)$ \\
\hline
\end{tabular}

*Data is presented as mean \pm standard deviation. BUN: blood urea nitrogen; CAP: community-acquired pneumonia; CRP: C-reactive protein;

HCAP: healthcare-associated pneumonia; $\mathrm{PaO}_{2}$ : partial pressure of oxygen in arterial blood

HCAP (24.5\%). Patients with HCAP were older and had a higher performance status than those with CAP. There were more cases of chronic heart failure, cerebrovascular disease and chronic kidney disease among patients with HCAP. In addition, the incidence of chronic respiratory failure was higher in patients with HCAP, although this difference was not statistically significant. The 30 -day mortality rate was higher $(19.0 \%$ vs. $10.1 \% ; p=0.11)$ and treatment failure was less frequently seen $(1.4 \%$ vs. $5.3 \%$; $\mathrm{p}=0.47)$ among patients with HCAP than among patients with CAP. However, these observations were not statistically significant.

Microbes were identified in 106 patients - 80 (35.1\%) patients from the CAP group $(\mathrm{n}=228)$ and $26(35.1 \%)$ from the HCAP group ( $\mathrm{n}=74$ ) (Table II). While the bacteriology of the patients in the two groups was similar, a significantly higher number of patients with HCAP than those with CAP were positive for Pseudomonas aeruginosa ( $\mathrm{n}=5$ vs. $\mathrm{n}=0$; 
Table II. Pathogens detected in the sputum culture of patients with CAP and those with HCAP ( $n=106)$.

\begin{tabular}{|c|c|c|c|}
\hline \multirow[t]{2}{*}{ Pathogen } & \multicolumn{2}{|c|}{ No. of patients (\%) } & \multirow[t]{2}{*}{ p-value } \\
\hline & $\begin{array}{c}\text { CAP } \\
(n=80)\end{array}$ & $\begin{array}{c}\text { HCAP } \\
(n=26)\end{array}$ & \\
\hline Haemophilus influenzae & $27(33.8)$ & $5(19.2)$ & NS \\
\hline \multicolumn{4}{|l|}{ Streptococcus pneumoniae } \\
\hline Penicillin-sensitive & $30(37.5)$ & $8(30.8)$ & NS \\
\hline Penicillin-intermediate & $5(6.3)$ & $3(11.5)$ & NS \\
\hline Penicillin-resistant & $1(1.3)$ & $0(0)$ & NS \\
\hline Klebsiella pneumonia & $3(3.8)$ & $3(11.5)$ & NS \\
\hline Moraxella catarrhalis & $12(15.0)$ & $2(7.7)$ & NS \\
\hline Staphylococcus aureus & & & NS \\
\hline Methicillin-resistant & $11(13.8)$ & $1(3.8)$ & \\
\hline Methicillin-sensitive & $0(0)$ & $0(0)$ & \\
\hline Pseudomonas aeruginosa & $0(0)$ & $5(19.2)$ & $<0.001$ \\
\hline Others & $7(8.8)$ & $0(0)$ & NS \\
\hline
\end{tabular}

NS: not significant

$\mathrm{p}<0.001$ ). Penicillin-resistant Streptococcus pneumonia (PRSP) was detected in patients with CAP, while MRSA was found in neither group.

The antibiotic treatments received by the patients are shown in Table III. Ceftriaxone was more frequently administered to patients with CAP than to those with HCAP $(74.6 \%$ vs. $39.2 \%$; $\mathrm{p}<0.0001)$. However, a greater number of patients with HCAP than those with CAP received piperacillin/tazobactam infusions $(32.4 \%$ vs. $8.3 \%$; p < 0.0001). Combination therapy with macrolide was administered to more patients with CAP $(20.6 \%$ vs. $8.1 \%$; $p<0.0001)$. Penicillin $G$ was equally administered to both groups (1.8\% vs. $1.4 \%)$ and was not related to 30 -day mortality.

For patients with CAP, all of the prognostic guidelines showed a rise in the mortality rate with an increase in severity score. However, patients with HCAP who were categorised as mild or moderate by A-DROP (i.e. scores 1-2) and CURB-65 (i.e. scores 1-2) resulted in 30-day mortality, while those categorised as mild or moderate by PSI and I-ROAD survived beyond 30 days. All the patients with HCAP who died had been categorised as moderate or severe by I-ROAD, and as severe or very severe by PSI (Table IV). On comparison of the severity scores between the two groups, all the four prognostic guidelines showed significantly higher scores in patients with HCAP (Table V).

To evaluate the prognostic accuracy of each set of guidelines, the patients were further divided into two groups, namely low-risk (i.e. A-DROP, 0-3; CURB-65, 0-2; I-ROAD, $0-2$; PSI, I-III) and high-risk (i.e. A-DROP $\geq 4$; CURB-65, $\geq 3$; I-ROAD, $\geq 3$; PSI, $\geq$ V) (Table VI). All the prognostic guidelines, with the exception of CURB-65, showed a high sensitivity in patients with CAP who were categorised into the high-risk group. The sensitivity of the I-ROAD and PSI scores was high among patients with HCAP. Although the severity scores of all the prognostic guidelines were able to predict 30-day mortality in patients with CAP (Fig. 2), I-ROAD exhibited a higher discriminatory power for patients with HCAP in the ROC curve (Fig. 3).
Table III. Antimicrobials administered in the initial therapy.

\begin{tabular}{|c|c|c|c|}
\hline \multirow[t]{2}{*}{ Antimicrobial } & \multicolumn{2}{|c|}{ No. of patients (\%) } & \multirow[t]{2}{*}{ p-value } \\
\hline & $\begin{array}{c}\text { CAP } \\
(n=228)\end{array}$ & $\begin{array}{l}\text { HCAP } \\
(n=74)\end{array}$ & \\
\hline \multicolumn{4}{|l|}{$\begin{array}{l}\text { Extended spectrum } \\
\text { cephalosporins }\end{array}$} \\
\hline Ceftriaxone & $170(74.6)$ & $29(39.2)$ & $<0.0001$ \\
\hline Cefepime & $10(4.4)$ & $7(9.5)$ & NS \\
\hline Cefotaxime & $5(2.2)$ & $1(1.4)$ & NS \\
\hline \multicolumn{4}{|l|}{$\begin{array}{l}\text { Betalactam/ } \\
\text { betalactamase-inhibitors }\end{array}$} \\
\hline Piperacillin/tazobactam & $19(8.3)$ & $24(32.4)$ & $<0.0001$ \\
\hline Ampicillin/sulbactam & $13(5.7)$ & $4(5.4)$ & NS \\
\hline Fluoroquinolone & $6(2.6)$ & $2(2.7)$ & NS \\
\hline Ciprofloxacin & $4(1.8)$ & $2(2.7)$ & NS \\
\hline Levofloxacin & $2(0.9)$ & $0(0)$ & NS \\
\hline \multicolumn{4}{|l|}{ Others } \\
\hline Meropenem & $1(0.4)$ & $1(1.4)$ & NS \\
\hline Penicillin G & $4(1.8)$ & $1(1.4)$ & NS \\
\hline Clindamycin & $1(0.4)$ & $1(1.4)$ & NS \\
\hline Vancomycin & $6(2.6)$ & $3(4.1)$ & NS \\
\hline Combination with macrolide & $47(20.6)$ & $6(8.1)$ & $<0.0001$ \\
\hline Combination with fluoroxoron & $4(1.8)$ & $3(4.1)$ & NS \\
\hline
\end{tabular}

NS: not significant

Table IV. Mortality according to the severity scores of the prognostic guidelines.

\begin{tabular}{|c|c|c|c|c|c|}
\hline \multirow[t]{2}{*}{ Guideline } & \multirow{2}{*}{$\begin{array}{c}\text { Severity } \\
\text { score }\end{array}$} & \multicolumn{4}{|c|}{ No. of patients (\%) } \\
\hline & & $\begin{array}{c}\text { CAP } \\
(n=228)\end{array}$ & $\begin{array}{c}\text { CAP } \\
\text { deaths }\end{array}$ & $\begin{array}{c}\text { HCAP } \\
(n=74)\end{array}$ & $\begin{array}{l}\text { HCAP } \\
\text { deaths }\end{array}$ \\
\hline \multirow[t]{6}{*}{ CURB-65 } & 0 & $25(11.0)$ & $0(0)$ & $1(1.4)$ & $1(100.0)$ \\
\hline & 1 & $79(34.6)$ & $4(5.1)$ & $8(10.8)$ & $0(0)$ \\
\hline & 2 & 71 (31.1) & 8 (11.3) & $20(27.0)$ & $3(15.0)$ \\
\hline & 3 & $38(16.7)$ & $6(15.8)$ & $30(40.5)$ & $5(16.7)$ \\
\hline & 4 & $14(6.1)$ & $4(28.6)$ & $14(18.9)$ & $5(35.7)$ \\
\hline & 5 & $1(0.4)$ & $1(100.0)$ & $1(1.4)$ & $0(0)$ \\
\hline \multirow[t]{6}{*}{ A-DROP } & 0 & 34 (14.9) & $0(0)$ & $0(0)$ & $0(0)$ \\
\hline & 1 & $51(22.4)$ & $2(3.9)$ & $9(12.2)$ & $1(11.1)$ \\
\hline & 2 & $69(30.3)$ & $3(4.3)$ & $17(23.0)$ & $1(5.9)$ \\
\hline & 3 & $41(18.0)$ & 8 (19.5) & $19(25.7)$ & $4(21.1)$ \\
\hline & 4 & $29(12.7)$ & $8(27.6)$ & $28(37.8)$ & $8(28.6)$ \\
\hline & 5 & $4(1.8)$ & $2(50.0)$ & $1(1.4)$ & $0(0)$ \\
\hline \multirow[t]{4}{*}{ I-ROAD } & 0 & $24(10.5)$ & $0(0)$ & $2(2.7)$ & $0(0)$ \\
\hline & Mild & $100(43.9)$ & $2(2.0)$ & $28(37.8)$ & $0(0)$ \\
\hline & Moderate & $42(18.4)$ & 5 (11.9) & $6(8.1)$ & 2 (33.3) \\
\hline & Severe & $62(27.2)$ & $16(25.8)$ & $38(51.4)$ & $12(31.6)$ \\
\hline \multirow[t]{5}{*}{ PSI } & 1 & $7(3.1)$ & $0(0)$ & $0(0)$ & $0(0)$ \\
\hline & II & $25(11.0)$ & $0(0)$ & $2(2.7)$ & $0(0)$ \\
\hline & III & $48(21.1)$ & $3(6.3)$ & $1(1.4)$ & $0(0)$ \\
\hline & IV & $90(39.5)$ & $5(5.6)$ & $35(47.3)$ & $2(5.7)$ \\
\hline & V & $58(25.4)$ & 15 (25.9) & $36(48.6)$ & 12 (33.3) \\
\hline
\end{tabular}

PSI: pneumonia severity index

\section{DISCUSSION}

In the treatment of CAP and HCAP, the question of which prognostic guideline is more useful remains controversial. For CAP, some reports have concluded that all prognostic 
Table V. Total number of deaths and mean severity scores of the two patient groups.

\begin{tabular}{lccc}
\hline Parameter & \multicolumn{2}{c}{ Mean \pm SD } & p-value \\
\cline { 2 - 3 } & $\begin{array}{c}\text { CAP } \\
(\mathbf{n}=\mathbf{2 2 8})\end{array}$ & $\begin{array}{c}\text { HCAP } \\
(\mathbf{n}=\mathbf{7 4})\end{array}$ & \\
\hline No. of deaths $(\%)$ & $23(10.1)$ & $14(18.9)$ & $<0.01$ \\
CURB-65 score & $1.7 \pm 1.1$ & $2.7 \pm 1.0$ & $<0.001$ \\
A-DROP score & $2.0 \pm 1.3$ & $2.9 \pm 1.1$ & $<0.001$ \\
I-ROAD score & $1.6 \pm 1.0$ & $2.1 \pm 1.0$ & $<0.001$ \\
PSI score & $112 \pm 40$ & $140 \pm 32$ & $<0.001$ \\
\hline
\end{tabular}

PSI: pneumonia severity index; SD: standard deviation

Table VI. Sensitivity, specificity, positive predictive value (PPV) and negative predictive value (NPV) of the prognostic guidelines according to patient group.

\begin{tabular}{lcccc}
\hline Guideline & Sensitivity & Specificity & PPV & NPV \\
\hline CAP group & & & & \\
CURB-65 & 47.0 & 79.5 & 47.8 & 79.5 \\
A-DROP & 96.9 & 88.9 & 43.5 & 88.8 \\
I-ROAD & 91.3 & 54.3 & 69.6 & 77.6 \\
PSI & 90.0 & 29.8 & 65.2 & 79.0 \\
HCAP group & & & & \\
CURB-65 & 71.4 & 41.7 & 71.4 & 41.7 \\
A-DROP & 57.1 & 65.0 & 57.1 & 65.0 \\
I-ROAD & 85.7 & 56.7 & 85.7 & 56.7 \\
PSI & 83.3 & 51.1 & 83.3 & 58.1 \\
\hline
\end{tabular}

Note: Data is presented as percentage. CAP: community-acquired pneumonia; HCAP: healthcare-associated pneumonia; PSI: pneumonia severity index

guidelines are similar and can correctly reflect the severity of pneumonia. ${ }^{(1,12)}$ However, a study by Higuchi et al documented that dehydration and some parameters, such as haematocrit and BUN, are not good prognostic factors for evaluating the severity of pneumonia in elderly patients. ${ }^{(13)}$ Higuchi et al also found that pneumonia in elderly patients categorised as mild or moderate by A-DROP showed a poor prognosis when comorbidities were present. ${ }^{(13)}$ Thus, it is reasonable to presume that there is an underestimation of the severity of pneumonia in elderly patients. Carrabba et al found that PSI and SCAP (severe community-acquired pneumonia) ${ }^{(14)}$ are useful prognostic tools for immunocompetent patients, with the former noted to be highly sensitive but poorly specific for the evaluation of HCAP severity in immunocompromised patients with HCAP. ${ }^{(15)}$ In a different study, Jeong et al demonstrated that the prognostic powers of PSI and CURB-65 for 30-day mortality were significantly lower for patients with HCAP than for patients with CAP. ${ }^{(16)}$ The findings of these studies suggest that the conventional prognostic guidelines used for CAP are not appropriate for the evaluation of HCAP severity. ${ }^{(15,16)}$

In the present study, we found that I-ROAD correctly reflected the severity of CAP and HCAP, whereas A-DROP and CURB-65 underestimated the severity of both. PSI was useful in evaluating the severity of CAP and HCAP, but there was no statistically significant difference between the two groups. Although PSI appeared to be as useful as I-ROAD for the evaluation of severity

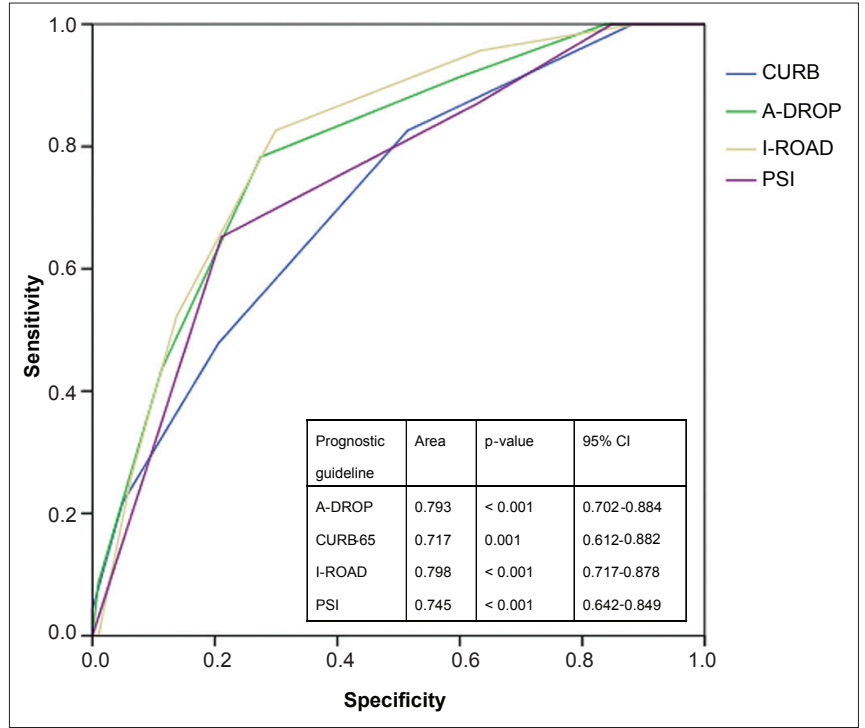

Fig. 2 Receiver-operating characteristic curve for the prediction of 30-day mortality for each score among high-risk patients with CAP.

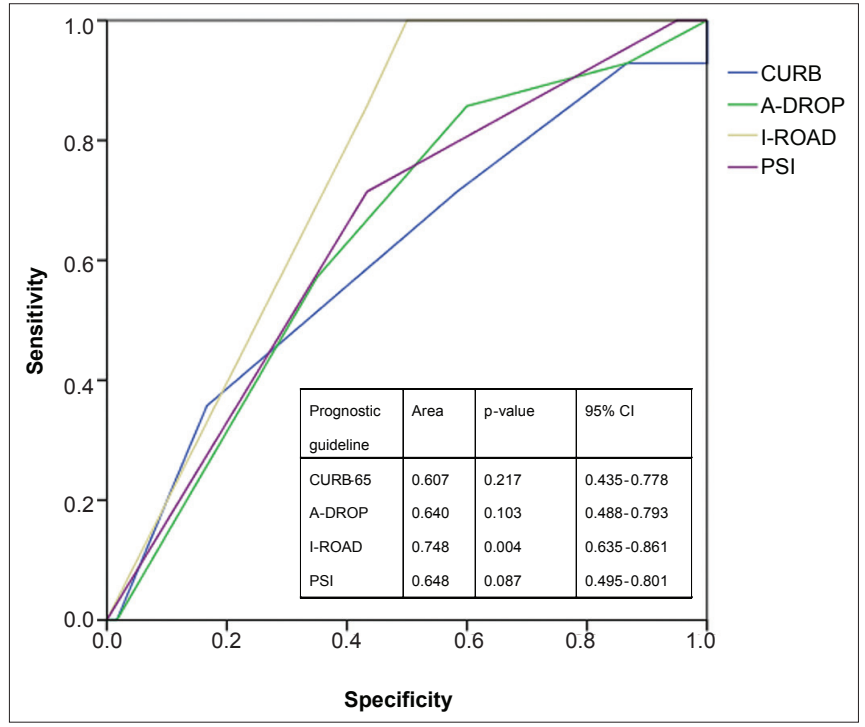

Fig. 3 Receiver-operating characteristic curve for the prediction of 30-day mortality for each score among high-risk patients with HCAP.

of CAP and HCAP, statistical significance was not found in the ROC curve.

In general, elderly patients aged over 70 years will tend to have comorbidities. ${ }^{(17)}$ Studies have shown that patients with HCAP are older and have more comorbidities than patients with CAP. ${ }^{(18-21)}$ When severity is assessed using A-DROP and CURB-65, these aforementioned factors could result in underestimation of the severity of pneumonia in elderly patients with CAP or HCAP, as both of these prognostic guidelines do not take into consideration the presence of comorbidities in their evaluation of the severity of pneumonia, unlike PSI and I-ROAD. However, as evaluation of the severity of pneumonia using PSI is time-consuming due to the complicated nature of its calculations, its use in Japan is limited. For the same reason, we do not recommend the use of PSI in the emergency room outpatient clinic. In terms of utility, I-ROAD is much more useful than PSI, and could 
possibly be more accurate than other prognostic guidelines for patients with HCAP. In the present study, although the sensitivity, specificity, and positive and negative predictive values of the I-ROAD score were found to be similar to those of the CURB-65, A-DROP and PSI scores, the I-ROAD score was significantly higher than the other scores in the ROC curve. Notwithstanding the strengths of I-ROAD and PSI, we emphasise that the prognostic accuracy of neither of these guidelines is high enough to be considered reliable. Therefore, such guidelines should only be viewed as one of many tools that can help physicians make a rational decision.

The present study found that patients with HCAP and those with CAP show different characteristics such as the rates of occurrence of MDR pathogens, initial treatment failure and 30-day mortality. Shindo et al reported the relationship between the rate of occurrence of MDR pathogens and initial treatment failure and inappropriate initial antibiotic treatment for HCAP. ${ }^{(18)}$ Jung et al showed via multivariate analysis that tube feeding and previous hospitalisation within 90 days of pneumonia onset were significant risk factors for frequency of occurrence of MDR pathogens. ${ }^{(20)}$

In the present study, the rate of occurrence of MDR pathogens among patients with HCAP $(15.0 \%)$ was relatively lower than that reported in other studies $(12.6 \%-38.1 \%) . .^{13,17-19)}$ Our rate of initial treatment failure for HCAP $(1.4 \%)$ was also much lower than that reported in other studies $(24.2 \%-35.9 \%){ }^{(16-19)}$ In contrast, the 30-day mortality rate among our patients with HCAP (18.9\%) was similar to that reported in other studies $(12 \%-29 \%){ }^{(18-21)}$

The contribution of MDR pathogens to the aetiology of HCAP and its empirical treatment, however, remains controversial. ${ }^{(19)}$ Ewig et al opined that the concept of HCAP contributes to confusion, potentially leading to overtreatment. ${ }^{(22)}$ In the present study, $50 \%$ of patients with HCAP received some antipseudomonal agent, but we could not assume that the antibiotic selections had contributed to the improvement in clinical outcome, even though the rates of occurrence of MDR pathogens, initial treatment failure and inappropriate antibiotic therapy among these patients were lower compared to other studies. ${ }^{(18-21)}$ The HCAP mortality rate worldwide and that reported in the present study are almost identical. Unfortunately, we could not determine the risk factors for HCAP mortality, as there was no clear relationship between HCAP mortality and the rates of occurrence of MDR pathogens or initial treatment failure. A prospective study should be performed to analyse and identify the risk factors, so that the mortality of patients with HCAP can be improved.

The present study was not without its limitations. First, as this was a retrospective study conducted on a very small population of patients, it may be less reliable with regard to the data collected, particularly for data such as physical examinations. A prospective study with a larger population will be necessary in order to verify the findings of the present study. Second, we did not discuss the possibility of atypical pneumonia, as the modality of diagnosis of atypical pneumonia is limited. No pathogens were detected in the cultures of $64.9 \%$ (196/302) of our study cohort. This suggests that atypical pneumonia, such as those caused by Mycoplasma pneumoniae or Chlamydophila pneumonia, is related to CAP and HCAP. Third, the nutritional status of the patients and the effects of tube feeding were not analysed in the present study. These factors may affect the rates of occurrence of MDR pathogens and mortality of patients with HCAP. ${ }^{(20,21)}$

In conclusion, the present study is the first of its kind to demonstrate the efficacy of I-ROAD in the evaluation of the severity of HCAP. We also found that I-ROAD has similar efficacy in assessing the severity of CAP. Based on our findings, I-ROAD appears to be more accurate for evaluating the severity of HCAP than other prognostic guidelines. Using I-ROAD to predict mortality could help clinicians make rational decisions regarding the best treatment and site of care for patients. A prospective study with a large sample size is needed to confirm our observations.

\section{ACKNOWLEDGEMENT}

We are grateful for the diligent and thorough critical reading of our manuscript by Mr John Wocher, Executive Vice President and Director of International Affairs/International Patient Services of Kameda Medical Center, Japan.

\section{REFERENCES}

1. Miyashita N, Matsushima T, Oka M; Japanese Respiratory Society. The JRS guidelines for the management of community-acquired pneumonia in adults: an update and new recommendations. Intern Med 2006; 45:419-28.

2. British Thoracic Society Standards of Care Committee. BTS guidelines for the management of community acquired pneumonia in adults. Thorax 2001; 56(Suppl 4):1-64.

3. Niederman MS, Mandell LA, Anzueto A, et al. Guidelines for the management of adults with community-acquired pneumonia: diagnosis, assessment of severity, antimicrobial therapy, and prevention. Am J Respir Crit Care Med 2001; 163:1730-54

4. American Thoracic Society, Infectious Disease Society of America. Guidelines for the management of adults with hospital-acquired, ventilatorassociated, and healthcare-associated pneumonia. Am J Respir Crit Care Med 2005; 171:388-416.

5. Japanese Respiratory Society. Establishment of new severity ratings based on analysis of hospital-acquired pneumonia. Respirology 2009; 14(Suppl 2): S4-9.

6. Seki M, Watanabe A, Mikasa K, Kadota J, Kohno S. Revision of the severity rating and classification of hospital-acquired pneumonia in the Japanese Respiratory Society guidelines. Respirology 2008; 13:880-5.

7. Zubrod CG, Sheiderman M, Frei E, et al. Appraisal of methods for the study of chemotherapy in man: Comparative therapeutic trial of nitrogen mustard and triethylene thiophosphoramide. J Chronic Dis 1960; 11:7-33.

8. Clinical and Laboratory Standards Institute: Performance Standards for Antimicrobial Susceptibility Testing; Eighteenth Informational Supplement. Vol 28. M100-S18. Wayne, Pennsylvania: CLSI, 2008.

9. Trouillet JL, Chastre J, Vuagnat A, et al. Ventilator-associated pneumonia caused by potentially drug-resistant bacteria. Am J Respir Crit Care Med 1998; 157:531-9.

10. Bradford PA. Extended-spectrum $\beta$-lactamases in the $21^{\text {st }}$ century: characterization, epidemiology, and detection of this important resistance threat. Clin Microbiol Rev 2001; 14:933-51.

11. Aujesky D, Auble TE, Yearly DM, et al. Prospective comparison of three validated prediction rules for prognosis in community-acquired pneumonia. Am J Med 2005; 118:384-92.

12. Usui K, Tanaka Y, Noda H, Ishihara T. [Comparison of three prediction rules for prognosis in community acquired pneumonia: Pneumonia Severity Index (PSI), CURB-65, and A-DROP]. Nihon Kokyuki Gakkai Zasshi 2009; 47:781-5. Japanese. 
13. Higuchi T, Ota K, Tanabe Y, Suzuki E, Gejyo F. [Severity classification and prognosis in hospitalized elderly patients with communityacquired pneumonia]. Nihon Ronen Igakkai Zasshi 2007; 44:483-9. Japanese.

14. Liapikou A, Ferrer M, Polverino E, et al. Severe community communityacquired pneumonia: validation of the Infectious Diseases Society of America/American Thoracic Society guidelines to predict an intensive care unit admission. Clin Infect Dis 2009; 48:377-85.

15. Carrabba M, Zarantonello M, Bonara $P$, et al. Severity assessment of healthcare-associated pneumonia and pneumonia in immunosuppression. Eur Respir J 2012; 40:1201-10.

16. Jeong $\mathrm{BH}$, Koh WJ, Yoo H, et al. Performances of prognostic scoring systems in patients with healthcare-associated pneumonia. Clin Infect Dis 2013 56:625-32.

17. Ministry of Health, Labour and Welfare, Insurance Department. Patients report. Tokyo, 2011. Available at: http://www.mhlw.go.jp/toukei/saikin/ hw/kanja/11/dl/toukei.pdf. Accessed March 10, 2013.

18. Shindo Y, Sato S, Maruyama E, et al. Health-care-associated pneumonia among hospitalized patients in a Japanese community hospital. Chest 2009; 135:633-40.

19. Grenier C, Pépin J, Nault V, et al. Impact of guideline-consistent therapy on outcome of patients with healthcare-associated and community-acquired pneumonia. J Antimicrob Chemother 2011; 66:1617-24

20. Sugisaki M, Enomoto T, Shibuya $Y$, et al. Clinical characteristics of healthcare-associated pneumonia in a public hospital in a metropolitan area of Japan. J Infect Chemother 2012; 18:352-60.

21. Jung JY, Park MS, Kim YS, et al. Healthcare-associated pneumonia among hospitalized patients in a Korean tertiary hospital. BMC Infect Dis 2011; $11: 61$

22. Ewig S, Welte T, Chastre J, Torres A. Rethinking the concepts of communityacquired and health-care-associated pneumonia. Lancet Infect Dis 2010; 10:279-87. 